\title{
Promoção da saúde mental das crianças: contributos dos enfermeiros
}

Promotion of children's mental health: nurses' contributions

Promoción de la salud mental de los niños: contribución de los enfermeros

\author{
Ernestina Maria Veríssimo Batoca Silva ${ }^{1}$ (i) https://orcid.org/0000-0002-4401-6296 \\ Daniel Silva ${ }^{1}$ ic hitps://orcid.orgy/0000-0003-2777-6688 \\ Graça Aparício ${ }^{1}$ io https://orcid.org/0000-0001-6149-062X \\ Isabel Bica ${ }^{1}$ io https://orcid.org/0000-0002-7019-0132 \\ Carlos Albuquerque ${ }^{1}$ io https://orcid.org/0000-0002-2297-0636 \\ Madalena Cunha ${ }^{1}$ io hitps://orcid.org/0000-0003-0710-9220
}

\section{Como citar:}

Silva EM, Silva D, Aparício G, Bica I, Albuquerque C, Cunha M. Promoção da saúde mental das crianças: contributos dos enfermeiros. Acta Paul Enferm. 2020;33:eAPE20180254.

DOI

http://dx.doi.org/10.37689/actaape/2020A00254

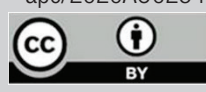

Descritores

Promoção da saúde; Saúde mental; Criança; Enfermagem pediátrica; Atenção primária à saúde.

Keywords Health promotion; Mental health; Child; Pediatric nursing; Primary health care

Descriptores

Promoción de la salud; Salud mental; Niño; Enfermería pediátric; Atención primaria de salud

\section{Submetido \\ 2 de Setembro de 2019 \\ Aceito \\ 20 de Dezembro de 2019}

Autor correspondente

Ernestina Maria Veríssimo Batoca da Silva E-mail: ernestinabatoca@gmail.com

\section{Resumo}

Objetivo: Descrever as intervenções de enfermagem para a promoção da saúde mental das crianças.

Métodos: Revisão integrativa da literatura realizada nas bases de dados PubMed, B-On e CINAHL utilizando os descritores "promoção da saúde", "saúde mental", "criança", "enfermagem" ou "papel do enfermeiro" ou "enfermagem pediátrica" ou "enfermagem de atenção primária", com a combinação dos operadores booleanos "AND" e "OR". Foram elegíveis artigos completos dos últimos 10 anos, nos idiomas português e inglês, coadunando aos critérios de inclusão e exclusão. Realizou-se análise qualitativa com a construção de três categorias.

Resultados: Incluíram-se quinze artigos que descreviam as intervenções de enfermagem com as crianças e adolescentes na escola, junto da família e com a comunidade, sendo que a maioria abordava as intervenções na escola com ênfase para a promoção da resiliência e comportamentos saudáveis.

Conclusão: Os estudos analisados evidenciam a importância dos enfermeiros na promoção da saúde mental das crianças em programas de educação em saúde mental, sensibilização e apoio às necessidades psicoafectivas, biológicas e sociais. Apresenta-se um leque de intervenções a serem organizadas e desenvolvidas pelos enfermeiros que trabalham na escola, com as famílias e em interligação com a comunidade.

\section{Abstract}

Objective: To describe nursing intervention to promote children's mental health.

Methods: This was an integrative review using PubMed, B-On, and CINAHL databases using the following keywords "health promotion", "mental health, "child", "nursing", or "role of nursing" or "pediatric nursing" or "primary nursing care", with combination of Boolean operators "AND" and "OR". Full-text articles published in Portuguese and English within 10 years were included, after application of inclusion and exclusion criteria. A qualitative analysis with construction of three categories were included.

Results: We included 15 articles describing nursing interventions for children and adolescents at school, for family and community. The majority of interventions were approach at school with an emphasis on promotion of resilience and a healthy behavior.

Conclusion: The analyzed studies showed the importance of nurses to promote children's mental health in mental health education programs, sensibilization and support to psychoaffective, biologic and social needs. A large variety of interventions organized and developed by nurses were observed at school, with families and as well as in the interrelationship with community. 


\section{Resumen}

Objetivo: Describir las intervenciones de enfermería para la promoción de la salud mental de los niños.

Métodos: Revisión integradora de la literatura realizada en las bases de datos PubMed, B-On y CINAHL utilizando los descriptores "promoción de la salud", "salud mental", "niño", "enfermería" o "papel del enfermero" o "enfermería pediátrica" o "enfermería de atención primaria", con la combinación de los operadores booleanos "AND" y "OR". Se incluyeron artículos completos de los últimos 10 años en los idiomas portugués e inglés, junto con los criterios de inclusión y exclusión. Se realizó un análisis cualitativo con la construcción de tres categorías.

Resultados: Se incluyeron 15 artículos que describían las intervenciones de enfermería con niños y adolescentes en la escuela, junto con la familia y la comunidad, y la mayoría abordaba las intervenciones en la escuela con énfasis en la promoción de la resiliencia y comportamientos saludables.

Conclusión: Los estudios analizados demuestran la importancia de los enfermeros en la promoción de la salud mental de los niños en programas de educación en salud mental, sensibilización y apoyo a las necesidades psicoafectivas, biológicas y sociales. Se presenta un abanico de intervenciones que los enfermeros que trabajan en escuelas deben organizar y llevar a cabo, junto con las familias e interconectados con la comunidad.

\section{Introdução}

Cada vez um maior número de crianças experiencia dificuldades em responder aos desafios desenvolvimentais que enfrentam e sofrem os efeitos negativos dos transtornos mentais. A prevalência dos transtornos mentais entre as crianças aumentou nos últimos anos e estima-se que, atualmente, 10 a $20 \%$ das crianças tenham um ou mais problemas de saúde mental. ${ }^{(1)}$ Para além das crianças com um diagnóstico de transtorno mental, existem muitas outras que evidenciam problemas de comportamento e que embora estejam abaixo dos limites do diagnóstico clínico, apresentam igualmente dificuldades ao nível da aprendizagem, da atenção e da instabilidade psicomotora, problemas relacionais, assim como manifestações de sofrimento emocional. ${ }^{(2)}$

Esta realidade exige um aumento da capacidade de resposta e o desenvolvimento de formas eficazes para atender as necessidades destas crianças e suas famílias, o mais precocemente possível. O Programa Nacional para a Saúde Mental publicado em Portugal privilegia um conjunto de estratégias de prevenção e promoçáo da saúde mental, entre as quais, programas de educação em saúde mental na idade escolar, prevenção da violência e do abuso de drogas ou programas de desenvolvimento pessoal e social. ${ }^{(3)}$ Relativamente às situaçóes de risco, à ocorrência de maus tratos ou à presença de necessidades de saúde especiais, cabe aos cuidadores de saúde inseridos nos Programas Nacional de Saúde Infantil e Juvenil, Acção de Saúde Crianças e Jovens em Risco, Nacional de Saúde Escolar e no Sistema Nacional de Intervenção Precoce na Infância, desenvolverem estratégias de prevenção. Os enfermei- ros integram cada um destes programas e têm sido reconhecidos como líderes de estratégias de promoção da saúde pela Organização Mundial de Saúde tendo contribuído para o conhecimento e literacia em saúde da população, entendida como a capacidade para tomar decisóes informadas sobre a saúde, mediante diversas abordagens, que se entrelaçam e que, juntas, são direcionadas para o desenvolvimento de ambientes, relaçôes, sentimentos e atitudes saudáveis e positivos. ${ }^{(4,5)}$

É reconhecido que os enfermeiros são frequentemente os profissionais mais próximos da escola e da comunidade e estão numa posição única para ajudar a identificar os problemas iniciais e oferecer intervenção precoce para diminuir as morbidades psicossociais nas crianças. ${ }^{(6,7)}$ Ao nível do Programa Nacional de Saúde Escolar de Portugal ao intervirem no jardim-de-infância e nas escolas do ensino básico e secundário os enfermeiros integrando as equipas de saúde escolar, assumem um papel ativo na gestão dos determinantes da saúde da comunidade educativa, nomeadamente saúde mental e emocional, educação alimentar, atividade física, saúde oral, cuidados posturais e prevenção de problemas musculoesqueléticos, mobilidade segura e prevenção de acidentes, educação ambiente e saúde, promoção dos afetos e da educação para a sexualidade, prevenção do consumo de tabaco, álcool e outras substâncias psicoactivas, prevenção de comportamentos aditivos sem substância (jogo, internet e outros). ${ }^{(2,8)}$ No relatório de 2014/2015 verificou-se que o maior investimento foi feito junto dos alunos do $1 .^{\circ}$ ciclo do ensino básico e dos educadores de infância do pré-escolar. ${ }^{(8)}$ Investir em promoção da saúde na escola é dar prioridade aos docentes, capacitando-os 
para que os determinantes da saúde sejam integrados nos currículos das mais diversas áreas do saber e a sua transmissão aos alunos seja pedagogicamente adequada ao nível de desenvolvimento do grupo escolar. Neste relatório o grande investimento da saúde escolar foi na melhoria da literacia para a saúde dos alunos, nomeadamente por meio de mais informação nas áreas da alimentação saudável, da educação para os afetos e a sexualidade, bem como para as questóes ambientais que comprometem a saúde. Contudo, a saúde escolar inclui também aspetos mais específicos direcionados a promover e proteger a saúde física e mental das crianças, pois o bem-estar mental e físico é vital para o desempenho académico do aluno. ${ }^{(9)}$ Os enfermeiros da saúde escolar são profissionais confiáveis e encontram-se numa posição privilegiada para desenvolver atividades de promoção da saúde e prevenção da doença, para reconhecer precocemente os sinais em crianças emocionalmente stressadas e dar apoio em situaçôes de crise. ${ }^{(9)}$

O Programa Nacional de Saúde Infantil e Juvenil português dá enfoque às questôes relacionadas com o desenvolvimento infantil e inclui como uma das principais linhas de atuação o investimento na prevenção das perturbações emocionais e do comportamento. ${ }^{(1)} \mathrm{Na}$ consulta de vigilância de saúde infantil e juvenil os enfermeiros assumem um papel importante $e^{(1)}$ na promoção da saúde, prevenção de doenças, identificação precoce e intervenção com crianças, adolescentes e suas famílias em risco para problemas psicossociais, proporcionando acompanhamento e apoio na superação de dificuldades. ${ }^{(5,6)}$ $\mathrm{O}$ apoio ao papel parental constitui um foco relevante em enfermagem no sentido de serem os pais os principais prestadores de cuidados e de assumirem como direito e dever a educação dos filhos e serem os seus modelos de referência para os filhos. De facto, a promoção da saúde ultrapassa o estilo de vida saudável, na direção do bem-estar global, não sendo responsabilidade exclusiva dos serviços de saúde. A mãe, o pai ou ambos, aliados ao profissional de saúde, devem comprometer-se em cuidar do filho, estando este sadio ou doente. Acredita-se que a incidência dos problemas psicossociais é subestimada devido a fatores como a identificação de problemas inadequada por profissionais dos serviços de saúde primários e mesmo o diagnóstico erróneo de doenças físicas que são sintomas psicossomáticos ou psiquiátricos. ${ }^{(6)}$ A promoção da saúde mental da criança exige a intervenção de vários técnicos, detendo os enfermeiros um papel importante quer na escola, na família e na comunidade e cujo foco deve ser direcionado para a dimensão holística que engloba os domínios biológico, psicológico e social. ${ }^{(10)}$ Enfermeiros que para promover a saúde mental das crianças e suas famílias assumem um contrato social e profissional mais amplo e que envolve a equidade e a justiça. ${ }^{(11)}$ Face ao exposto, este estudo tem como objetivo descrever as intervençóes de enfermagem para a promoção da saúde mental das crianças. Destaca-se a pertinência de sistematizar evidências sobre as intervençóes de enfermagem que consistem na ação tomada em resposta a um diagnóstico de enfermagem de modo a produzir um resultado de enfermagem. ${ }^{(12)}$ As ações de enfermagem descritas nos estudos analisados incluem os seguintes enunciados: identificar, encorajar, incentivar, promover, ensinar, apoiar, aconselhar, colaborar, estabelecer relação. A finalidade deste artigo é contribuir para a melhoria da qualidade de cuidados de enfermagem nesta área.

\section{Métodos}

Para a elaboração da revisão integrativa percorremos as etapas da identificaçáo do tema, definiçáo da questão de pesquisa, estabelecimento de critérios de inclusão e exclusão dos estudos, identificação dos estudos selecionados, avaliação dos estudos incluídos, análise e interpretação dos resultados e apresentação da síntese da revisão do conhecimento. ${ }^{(13)}$ Partiu-se da seguinte questão norteadora: "Quais são as intervençôes de enfermagem para promover a saúde mental das crianças, descritas na literatura?" Para a coleta dos artigos, realizou-se a busca online, em maio de 2019, nas bases de dados National Library of Medicine and National Institutes of Health - PubMed, B-On e Cumulative Index to Nursing and Allied Health Literature - CINAHL. O levantamento realizado utilizou os termos iden- 
tificados nos descritores Ciências da Saúde (DeCS) e no Medical Subject Headings (Mesh). A pesquisa foi por assunto (subject) e a estratégia de busca foi a seguinte: "health promotion" AND "mental health" AND "child" AND ("nursing" OR "nurse's role" OR "pediatric nursing” OR "primary care nursing"). A busca e seleção dos estudos foram feitas por dois pesquisadores, de forma independente. Os critérios de inclusão foram: artigos que apresentassem intervençóes de enfermagem para a promoção da saúde mental de crianças nos idiomas português ou inglês, publicados entre 2009 e 2019. Optouse por incluir estudos dos últimos dez anos para se obter um maior corpus de estudos. Para a selecção dos estudos seguimos as recomendaçóes do método Preferred Reporting Items for Systematic reviews and Meta-Analyses - PRISMA ${ }^{(13)}$ (Figura 1). Assim, os artigos foram inicialmente selecionados pelo título e resumo e, posteriormente, lidos na íntegra, incluindo-se os que continham intervençôes de enfermagem para promover a saúde mental das crianças. Os artigos repetidos em mais de uma base de dados foram analisados uma única vez.

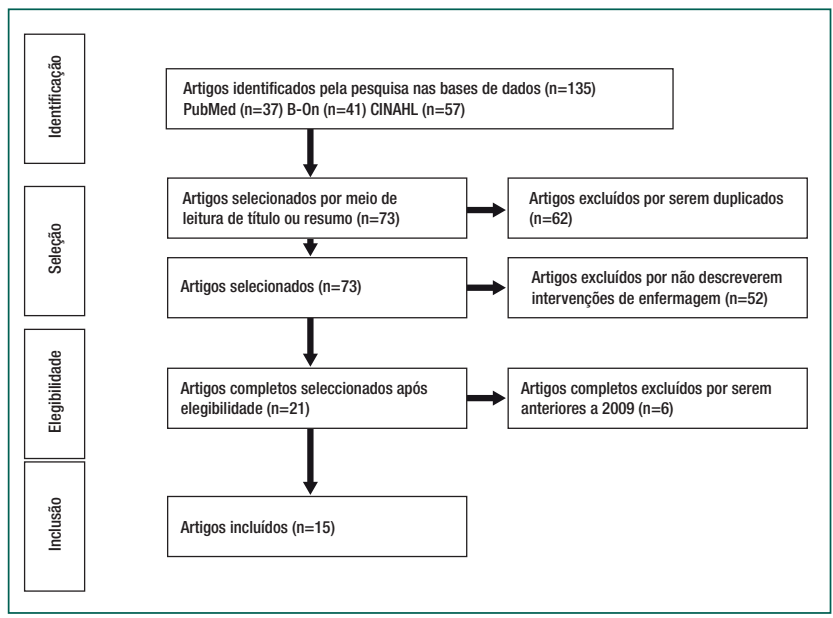

Figura 1. Fluxograma do processo de identificação, seleção e inclusão dos artigos, adaptado do PRISMA

Os artigos foram analisados considerando os critérios estabelecidos, isto é, serem projetos ou investigações que apresentassem intervençôes de enfermagem para a promoção da saúde mental das crianças. Apesar dos estudos também apresentarem resultados sobre adolescentes, o interesse foi apenas com as crianças. A classificação do nível de evidências (NE) foi a seguinte:
Nível I, revisão sistemática ou metanálise; Nível II, ensaio clínico controlado randomizado; Nível III, ensaio clínico controlado sem randomização; Nível IV, estudos de coorte ou caso-controle bem delineados; Nível $\mathrm{V}$, revisão sistemática de estudos qualitativos e descritivos; Nível VI, estudos descritivos ou qualitativos; e Nível VII, opiniāo de autoridades ou relato de especialistas. ${ }^{(14)}$ Após a leitura dos artigos procedeu-se à análise qualitativa e organização dos dados apresentados nos resultados e de acordo com as seguintes categorias: intervençóes de enfermagem na escola, intervençóes de enfermagem junto da família e intervenções de enfermagem na comunidade.

\section{Resultados}

Dos quinze artigos incluídos, obtidos nas bases de dados electrónicas referidas, todos foram publicados em inglês. ${ }^{(10,11,15-27)}$ Quanto ao país de realização seis são dos Estados Unidos da América, ${ }^{(10,11,20,24,25,27)}$ seis do Reino Unido, ${ }^{(15-18,21,22)}$ dois da Austrália ${ }^{(19,23)}$ e um do Canadá. ${ }^{(26)}$ No que se refere ao ano de publicação, um foi publicado em 2009, ${ }^{(15)}$ três em $2010,{ }^{(22-24)}$ um em 2011, ${ }^{(18)}$ um em 2012, ${ }^{(10)}$ um em 2013,(16) dois em 2014,(21,27) três em 2015, ${ }^{(11,20,25)}$ dois em 2017,(19,26) e um em 2018. (17) Quanto ao $\mathrm{NE}$, três artigos enquadram-se no nível $\mathrm{V}_{,}^{(10,20,27)}$ um no nível VI ${ }^{(15)}$ e onze no nível VII. ${ }^{(11,16-19,21-26)}$ Constatou-se que treze são direcionadas à esco$\mathrm{la}^{(10,11,15-25)}$ e sete se inserem em mais de uma das três categorias identificadas (Quadro 1). ${ }^{(10,11,17,21,23,24,26)}$

\section{Discussão}

Os resultados da produção científica desta revisão integrativa estiveram relacionados com as intervençôes de enfermagem para a promoção da saúde mental das crianças. Evidenciaram a identificação de problemas psicoafectivos, biológicas e sociais, a educação para a saúde e bem-estar e, ainda, o apoio e encaminhamento das crianças e famílias nas suas necessidades. Este estudo apresenta como limitação a busca em três bases de dados eletrônicas, tendo em vista a possibilidade de estudos indexados em 
Quadro 1. Intervenções de enfermagem para a promoção da saúde mental das crianças a serem desenvolvidas na escola, junto da família, na comunidade e classificação dos artigos por nível de evidência

\begin{tabular}{|c|c|}
\hline Intervenções de enfermagem na escola & Nível de Evidência \\
\hline Deteção precoce de problemas de saúde mental das crianças e adolescentes, incentivando-os a falar mais sobre os seus sentimentos. & $\begin{array}{c}V \mid(15) \\
V \mid I^{(16,17) \mid}\end{array}$ \\
\hline $\begin{array}{l}\text { Promoção do desenvolvimento da resiliência emocional das crianças e adolescentes: Incentivo a ter pensamento positivo, ser optimista, abraçar os desafios, a fazer algo para } \\
\text { ajudar outra pessoa, a brincar e a desenvolver criatividade, a promover a auto-estima e auto-eficácia, a desenvolver bons relacionamentos e empatia entre os pares, a explorar } \\
\text { as oportunidades de autodescoberta, de resolução dos problemas e aprender com eles e a desenvolver um senso do certo e errado. }\end{array}$ & $\begin{array}{c}\left.\mathrm{V}\right|^{(15)} \\
\mathrm{VI}(11,18,19)\end{array}$ \\
\hline Promoção e incentivo a uma alimentação saudável, reduzindo a obesidade, a dormir bem e fazer exercícios regularmente. & $\begin{array}{l}V^{(10,20)} \\
V\|\|^{(17,18)}\end{array}$ \\
\hline $\begin{array}{l}\text { Promoção da saúde emocional e bem-estar com fortalecimento da participação da comunidade, melhorando a inclusão social e reduzindo o estigma associado com } \\
\text { problemas de saúde mental. }\end{array}$ & $V \mid \|^{(18,21)}$ \\
\hline Promoção da saúde e do bem-estar de todos os rapazes e meninos, contribuindo para o desenvolvimento de políticas e práticas sensíveis às necessidades distintas ao género. & $V \|^{(22)}$ \\
\hline $\begin{array}{l}\text { Promoção do conhecimento cognitivo, histórico, cultural e experimental dos alunos, tratando os jovens como conhecedores, convidando-os a compartilhar a sua opinião e } \\
\text { testar ideias em um ambiente de apoio. }\end{array}$ & $V \mid \|^{(19)}$ \\
\hline $\begin{array}{l}\text { Fornecimento de oportunidades para os jovens serem pró-ativos e garantir a saúde mental e o bem-estar, ampliando o repertório de habilidades positivas que possam gerar } \\
\text { soluções para o enfrentamento de emoções e situações estressantes. }\end{array}$ & $\begin{array}{l}V^{(10)} \\
V I I^{(19)}\end{array}$ \\
\hline Encorajamento dos jovens a se unirem como um grupo e estabelecer formas compartilhadas de trabalho com os professores e a comunidade. & $V I I^{(19)}$ \\
\hline Envolvimento em atividades em que se sintam ouvidos e valorizados, construam a autoconfiança, melhorem o autocuidado e o cuidar dos outros. & VIII(17,19) \\
\hline Promoção da abordagem autogénica - o cultivo do bem-estar, baseado em explorações de alegria e em desenvolver fatores de proteção e não apenas em evitar riscos. & $V I^{(19)}$ \\
\hline $\begin{array}{l}\text { Incentivo a sair da zona de conforto com confiança para novas áreas. Empoderamento dos alunos, onde desenvolvem um lócus interno de controle, autoconfiança para } \\
\text { enfrentar desafios e à vontade para considerar ideias novas ou divergentes e assumir a responsabilidade por seu próprio comportamento. }\end{array}$ & $V I^{(19)}$ \\
\hline Apoio aos comportamentos saudáveis, ambientes escolares seguros e positivos e o envolvimento da família e da comunidade na escola. & $\begin{array}{l}\mathrm{V}^{(20)} \\
\mathrm{V} \|^{(23)}\end{array}$ \\
\hline Ensino de habilidades de emoções-sociais para as crianças e adolescentes. & $\begin{array}{l}V^{(10)} \\
V I I^{(24)}\end{array}$ \\
\hline $\begin{array}{l}\text { Encorajamento para o bem-estar emocional dos jovens com base na terapia cognitivo-comportamental: aconselhar estratégias práticas de auto-ajuda, ajudar a aumentar a } \\
\text { atividade física e a encontrar apoio social, monitorizar o impacto das mudanças de estilo de vida. }\end{array}$ & $\mathrm{Vl}^{(15)}$ \\
\hline Apoio com intervenções breves dirigidas à higiene pessoal, atendimento odontológico, alimentação saudável, estilos de vida, saúde emocional, sono e rotinas. & $V \mid \|^{(17,25)}$ \\
\hline Informação, suporte e conselhos aos jovens através dos media tais como Facebook ou Twiter. & $V \mid I^{(17)}$ \\
\hline \multicolumn{2}{|l|}{ Intervenções de enfermagem junto da família } \\
\hline $\begin{array}{l}\text { Identificação de problemas na saúde das crianças e desenvolvimento (como dificuldades de aprendizagem), segurança (como a negligência dos pais) e auto-suficiência } \\
\text { económica dos pais fornecendo ajuda o mais cedo possível. }\end{array}$ & $V \|^{(21)}$ \\
\hline $\begin{array}{l}\text { Incentivo a uma boa saúde e bem-estar ao longo da vida, iniciando na gravidez e incluindo visita domiciliária desde a gravidez precoce até aos dois anos de idade e } \\
\text { planeamento das necessidades de saúde. }\end{array}$ & VIII21,24,26) \\
\hline Apoio aos pais nas crises e ajudá-los a estabelecer relacionamentos e ambiente saudáveis através do qual a criança pode crescer. & $V \mid I^{(11)}$ \\
\hline Incentivo às mães a amamentar. & $V \|^{(21)}$ \\
\hline $\begin{array}{l}\text { Ajuda aos pais a desenvolver um vínculo forte com as crianças }{ }^{(20)} \text {. Apoio nos relacionamentos entre pais e filhos e proporcionando abordagens individualizadas para as } \\
\text { necessidades de cada família. }\end{array}$ & VII (17,24,26) \\
\hline Fornecimento de cuidados centrados na pessoa e dirigidos para o desenvolvimento de relações positivas mãe-bebé. & $V \|^{(26)}$ \\
\hline $\begin{array}{l}\text { Apoio nas necessidades das famílias ajudando a criar uma imagem mais positiva de pais e do ambiente que eles proporcionam para o crescimento e desenvolvimento dos } \\
\text { filhos. }\end{array}$ & $V\|\|^{(24)}$ \\
\hline $\begin{array}{l}\text { Aconselhamento de estratégias de pensamento positivo com as mães e que promovam a sua própria saúde mental e reduzam o risco de depressão e stressores crónicos } \\
\text { evitando o impacto que tem sobre a criança. }\end{array}$ & $V \|^{(24)}$ \\
\hline Proteção das crianças de doenças graves, através de triagem e imunização. & $V \|^{(21)}$ \\
\hline Encaminhamento das mães e crianças de famílias de baixos recursos económicos. & $V \|^{(21)}$ \\
\hline Capacitação dos pais a criar uma vida melhor para seus filhos e para si mesmos. & $V \|^{(26)}$ \\
\hline \multicolumn{2}{|l|}{ Intervenções de enfermagem na comunidade } \\
\hline $\begin{array}{l}\text { Avaliação e referenciação das desigualdades sociais, adequando as intervenções em direção à equidade em saúde, desenvolvendo parcerias com outros sectores. } \\
\text { Participação no desenvolvimento de políticas de inclusão de grupos vulneráveis. }\end{array}$ & $V \|^{(26)}$ \\
\hline Estabelecimento de relação com as escolas, assumindo um papel ativo da liderança da equipe oferecendo apoio de enfermagem e supervisão da gestão. & VIII(17,23) \\
\hline $\begin{array}{l}\text { Fornecimento de apoio de saúde e bem-estar em unidades móveis: vacinação, sessões de educação para a saúde sobre temas como álcool e fumar, alimentação saudável, } \\
\text { exercício físico e higienização das mãos. }\end{array}$ & $V \mid I^{(17)}$ \\
\hline Visitação domiciliária e educação da família. & $\begin{array}{l}V^{(10)} \\
V\|\|^{(24)}\end{array}$ \\
\hline Recomendação a exposição a espaços verdes - jardins, parques, florestas, openspaces, hortas terapêuticas, comunhão com a natureza, cam & $V^{(27)}$ \\
\hline
\end{tabular}

outros locais. Além disso, a inclusão de estudos dos últimos dez anos aumentou a possibilidade de dados menos recentes, contudo isso se fez necessário para se obter um maior corpus de estudos.

Como contribuição para a melhoria da qualidade de cuidados de enfermagem, esta revisão traz subsídios para que o enfermeiro intervenha na promoção da saúde mental das crianças. Nesse sentido, os artigos reportam relativamente à escola o detetar precoce de problemas, ${ }^{(15-17)}$ o promover o bem-estar e o fortalecer as capacidades de resiliência ao longo da vida, incentivar a desenvolver bons relaciona- 
mentos e empatia entre os pares, ${ }^{(11,15,18,19)}$ incentivar a explorar as oportunidades de autodescoberta, de resolução dos problemas e aprender com eles. ${ }^{(10,19)}$ Foi também evidenciado o incentivar a envolver as crianças em atividades em que elas se sintam ouvidas e valorizadas, ${ }^{(17,19)}$ o ensinar habilidades de emoçôes-sociais ${ }^{(10,24)}$ e o proporcionar intervençôes breves com apoio à higiene pessoal, atendimento odontológico, alimentação saudável, estilos de vida, saúde emocional, sono e rotinas. ${ }^{(17,25)} \mathrm{Na}$ escola estão reunidas oportunidades para aprender e praticar comportamentos saudáveis, pelo que emerge o fornecer serviços de saúde escolar, criando ambientes escolares seguros e positivos e envolvendo as famílias e a comunidade na escola ${ }^{(20,23)}$ contribuindo para melhorar a inclusão social. ${ }^{(18,21)}$ Nesse sentido, salienta-se a importância dos enfermeiros realizarem formação e treino para serem capazes de identificar o sofrimento psíquico em crianças e adolescentes e intervirem adequadamente. ${ }^{(28)}$

É importante igualmente intervir precocemente na família pois os problemas de saúde mental das crianças e adolescentes têm muitas vezes a sua origem na infância e no período pré-natal pelo que se deve incentivar uma boa saúde e bem-estar ao longo da vida, iniciando na gravidez e incluindo visita domiciliária desde o início da gravidez até aos dois anos de idade da criança e o planeamento das necessidades de saúde. ${ }^{(21,24,26)}$ Para promover a saúde mental das crianças deve-se ajudar os pais a desenvolver um vínculo forte com as crianças e incentivar os cuidados que mantêm as crianças saudáveis e seguras. ${ }^{(21)}$ Em complemento importa capacitar os pais a criar uma vida melhor para seus filhos e para si mesmos ${ }^{(26)}$ e apoiar as famílias de baixos recursos económicos. ${ }^{(21)}$ Nesta categoria sobressaem intervençôes para incentivar as relaçóes interpessoais positivas entre os membros da família e o capacitar as famílias no cumprimento das suas funçôes básicas favorecedoras do crescimento e desenvolvimento dos filhos. ${ }^{(24)}$ Assim, reconhece-se que a capacidade dos enfermeiros para trabalhar em parceria com os pais é uma competência essencial. ${ }^{(29)}$ Os pais devem ser vistos pelos enfermeiros como parceiros no cuidar da criança, promovendo as competências parentais para prestar cuidados, reduzir o nível de stress associado ao papel parental e preparar os pais para promover a autonomia da criança.

A comunidade tem grande impacto na promoção da saúde mental dos bebés, crianças e adolescentes pelo que os estudos que integraram esta revisão apontam como intervençóes de enfermagem o fortalecer a participação da comunidade. Respeitando as especificidades culturais da comunidade deve-se apoiar estratégias de promoção da saúde mental para as crianças, começando por avaliar e reportar as desigualdades e orientar em direção à equidade em saúde, através de parcerias com outros setores. (26) Outras importantes intervenções de enfermagem consistem em fazer visitação domiciliária, educação da família ${ }^{(10,24)}$ e fornecer apoio de saúde e bem-estar em unidades móveis ${ }^{(17)}$ (constituídas por uma viatura equipada para prestar cuidados de saúde em proximidade). Sabemos que a saúde mental considera como as circunstâncias biológicas, psicológicas e sociais se podem cruzar e interagir ao longo da vida para afetar trajetórias de saúde e doença, pelo que importa intervir nas crianças, de forma planeada e articulada em prol do futuro.

\section{Conclusão}

Face ao grande número e pertinentes intervenções de enfermagem para promover o bem-estar emocional, associado à boa saúde física e bem-estar geral das crianças e adolescentes, concluímos que este artigo traz subsídios para repensar e estruturar as intervençóes para promover a sua saúde mental na escola, junto da família e na comunidade. Realça-se a importância de intervenções psicoeducativas com ênfase no ensino, no aconselhamento, no incentivo à adoção de comportamentos saudáveis, no esclarecimento de dúvidas às crianças, adolescentes e pais, além de especial atenção às interaçóes com a comunidade no desenvolvimento de parcerias, visitas domiciliares e atividades ao ar livre. Além disso, constatou-se que os enfermeiros têm um papel alargado que passa por detetar problemas, interromper trajetórias de desenvolvimento negativo, promover comportamentos e estilos de vida saudáveis e melhorar a inclusão social com fortalecimento da participação da comunidade. 


\section{Agradecimentos}

Os nossos agradecimentos ao Fundo Social Europeu, ao Programa Operacional CENTRO 2020 e à Fundação para a Ciência e Tecnologia do Governo Português, instituiçóes públicas que se constituem como fontes de financiamento do Projeto de Investigação MAISaúde Mental "Monitorização e avaliação dos indicadores de saúde mental das crianças e adolescentes: Da investigação à prática" (código de identificação: CENTRO-01-0145FEDER-023293), onde este estudo se integra.

\section{Referências}

1. Portugal. Ministério da Saúde. Direção-Geral da Saúde. Programa nacional de saúde infantil e juvenil. Lisboa: DGS; 2013.

2. Portugal. Ministério da Saúde. Direção-Geral da Saúde. Programa nacional de saúde escolar 2015 [Internet]. Lisboa: DGS; 2016. [citado 2018 Dez 4]. Disponível em: https://observatoriolisboa.eapn.pt/ficheiro/Programa-Nacional-de-Sa\%C3\%BAdeEscolar-2015.pdf.

3. Portugal. Ministério da Saúde. Direção-Geral da Saúde. Programa nacional para a saúde mental. Lisboa: DGS; 2017. [citado 2018 dez 4]. Disponível em: https://www.dgs.pt/em-destaque/relatorio-doprograma-nacional-para-a-saude-mental-2017.aspx

4. Bittencourt MN, Marques MI, Barroso TM. Contributions of nursing theories in the practice of the mental health promotion]. Rev Enf Ref. 2018; SER IV(18):125-32. Portuguese.

5. Vasconcelos VM, Frota MA, Martins MC, Machado MM. Puericultura em enfermagem e educação em saúde: percepção de mães na estratégia saúde da família. Esc Anna Nery. 2012;16(2):326-31.

6. Melnyk BM, Moldenhauer Z, Veenema T, Gullo S, McMurtrie M, O'Leary E, et al. The KySS (Keep your children/yourself Safe and Secure) campaign: a national effort to reduce psychosocial morbidities in children and adolescents. J Pediatr Health Care. 2001;15(2):31A-4A.

7. Sherwin S, Rose K. Meeting identified needs: innovation in practice, part 2. Br J Sch Nurse. 2014;9(10):492-5.

8. Portugal. Ministério da Saúde. Direção-Geral da Saúde. Relatório técnico. Programa nacional de saúde escolar: avaliação do ano letivo 2014/2015. [Internet]. Lisboa: DGS; 2016. [citado 2018 dez 4]. Disponível em: https://www.dgs.pt/documentos-e-publicacoes/ relatorio-tecnico-de-avaliacao-do-programa-nacional-de-saudeescolar-do-ano-letivo-20142015-pdf.aspx.

9. Davis-Alldritt L. Children's mental health: the unmet need. NASN Sch Nurse. 2013;28(2):66-7.
10. Zauszniewski JA, Bekhet A, Haberlein S. A decade of published evidence for psychiatric and mental health nursing interventions. Online J Issues Nurs. 2012;17(3):8.

11. Delaney KR. Why do we need a child psychiatric nursing specialty? J Child Adolesc Psychiatr Nurs. 2015;28(1):1-2.

12. Conselho Internacional de Enfermeiros. [International classification for nursing practice - CIPE® Version 2015]. Lisboa: Ordem dos Enfermeiros; 2016. Portuguese.

13. Moher D, Liberati A, Tetzlaff J, Altman DG; PRISMA Group. Preferred reporting items for systematic reviews and meta-analyses: the PRISMA statement. Int J Surg. 2010;8(5):336-41.

14. Melnyk BM, Fineout-Overholt E. Evidence-based practice in nursing \& healthcare: A guide to best practice. Philadelphia: Lippincott Williams \& Wilkins; 2011.

15. Day P. The use of CBT to strengthen emotional wellbeing. Br J Sch Nurse. 2009;4(3):130-2.

16. Bhardwa S. A vital resource in reducing mental health problems. $\mathrm{Br} \mathrm{J}$ Sch Nurse. 2013;8(8):406.

17. Meet the Doncaster and North Lincolnshire school nurses. Br J Sch Nurse. 2018;13(2):83-5.

18. McDougall T. Mental health problems in childhood and adolescence. Nurs Stand. 2011;26(14):48-56.

19. McAllister M, Knight BA, Withyman C. Merging contemporary learning theory with mental health promotion to produce an effective schoolsbased program. Nurse Educ Pract. 2017;25(25):74-9.

20. Michael SL, Merlo CL, Basch CE, Wentzel KR, Wechsler H. Critical connections: health and academics. J Sch Health. 2015;85(11):740-58.

21. Glasper A. Reinforcing the commitment to a healthy start for all children. Br J Nurs. 2014;23(6):336-7.

22. Peate I. The mental health of men and boys: an overview. $\mathrm{Br} \mathrm{J}$ Nurs. 2010;19(19):1231-5.

23. Woodhouse $A$. Is there a future in mental health promotion and consultation for nurses? Contemp Nurse. 2010;34(2):177-89.

24. Staten RT, Peden AR. Prevention column. J Child Adolesc Psychiatr Nurs. 2010;23(1):42-3.

25. National Association of School Nurses (NASN). School Based Health Centers. The complementary roles of the school nurse and schoolbased health centers (Position Statement). Silver Spring: NASN; 2015.

26. Marcellus L, Shahram SZ. Starting at the Beginning: The Role of Public Health Nursing in Promoting Infant and Early Childhood Mental Health. Nurs Leadersh (Tor Ont). 2017;30(3):43-53.

27. Flaskerud $\mathrm{JH}$. Communing with nature. Issues Ment Health Nurs. 2014;35(12):975-8.

28. Weaver C, Rogers K, Gomez M, Gilder R, Yoder-Wise PS. Implementing MH-TIPS: A Growing Need for Mental Health Services for School-Age Children. NASN Sch Nurse. $2019 ; 34(1): 25-8$.

29. Sousa PC. 0 exercício parental durante a hospitalização do filho: intencionalidades terapêuticas de enfermagem face à parceria de cuidados [tese]. Porto: Universidade Católica Portuguesa; 2012. 